\title{
RECURSOS CONTEMPORÂNEOS DO PLANEJAMENTO ESTÉTICO INTEGRADO
}

\author{
Danielle Nishitani Shibasaki Estudante de graduação da Escola Bahiana de \\ Medicina e Saúde Pública. \\ Vivian Leite Martins Estudante de graduação da Escola Bahiana de \\ Medicina e Saúde Pública. \\ Clara Lemos Leal Mestrado em Clínica Odontológica pela Escola \\ Bahiana de Medicina e Saúde Pública. Professora \\ assistente da Faculdade Regional da Bahia. \\ Ana Paula Vaz Queiroz \\ Estudante de graduação da Escola Bahiana de \\ Medicina e Saúde Pública. \\ Paula Mathias \\ Doutorado em Clínica Odontológica pela \\ Universidade Estadual de Campinas. Professora \\ associada da Universidade Federal da Bahia.
Andrea Nóbrega Cavalcanti Doutorado em Clínica Odontológica pela Universidade Estadual de Campinas. Professora adjunta da Escola Bahiana de Medicina e Saúde \\ Publica e da Universidade Federal da Bahia.
}

Endereço para correspondência: dani_shibasaki@hotmail.com

\begin{abstract}
Resumo
A busca por tratamentos estéticos vem crescendo e, nesse sentido, os pacientes comparecem cada vez mais aos consultórios para obter sorriso agradável. O objetivo deste trabalho é descrever as principais ferramentas utilizadas na execução de planejamentos estéticos. De acordo com o estudo da literatura sobre o assunto e com a aplicação prática destes princípios, verifica-se que fotografias, enceramentos diagnósticos e planejamentos digitais são ferramentas indispensáveis para uma avaliação detalhada de todos os fatores que interferem na harmonia e simetria dos elementos que compõem o sorriso, possibilitando assim, bons resultados.
\end{abstract}

Palavras-chave: Planejamento estético. Análise pré-operatória. Odontologia restauradora.

\section{CONTEMPORARY RESOURCES OF AESTHETIC INTEGRATED PLANNING: A CASE REPORT}

\begin{abstract}
The search for aesthetic treatments has increased and, accordingly, patients used to attend clinics for pleasant smile. The objective of this article is to describe the main tools used in the execution of schedules aesthetic. According to the study of literature on the subject and the practical application of these principles, it appears that photographs, waxing diagnostics and digital planning are indispensable tools for a detailed evaluation of all factors affecting the harmony and symmetry of the elements that compose smile, thus enabling good results.
\end{abstract}

Keywords: Aesthetic Planning. Analysis Pre-operative. Restorative dentisty. 


\section{INTRODUÇÃO}

A melhoria do sorriso por meio de procedimentos estéticos representa uma grande demanda da sociedade contemporânea, uma vez que a aparência física desempenha papel importante nas relações sociais, principalmente diante dos novos padrões de beleza, nos quais dentes brancos e alinhados são considerados de alta relevância. ${ }^{(1)}$

Excelência estética requer planejamento criterioso que envolva a avaliação de todos os fatores que interferem na harmonia e simetria dos elementos que compõem o sorriso. ${ }^{(2)}$ Neste sentido, é importante que haja um estudo detalhado de cada caso para que seja possível planejar individualmente os tratamentos, a fim de se obter bons resultados.

Desta maneira, este trabalho teve como objetivo realizar o planejamento estético de um caso clínico, utilizando os principais recursos tecnológicos e clínicos disponíveis para a previsão dos resultados e comunicação entre profissional, paciente e laboratório.

\section{RELATO DE CASO}

Para a execução do caso foi selecionada uma paciente, sexo feminino, 21 anos, com boa condição de saúde geral e bucal, queixando-se do tamanho e cor dos dentes e presença de diastemas entre os caninos e incisivos superiores.

A voluntária, ciente dos objetivos deste estudo, assinou o termo de consentimento livre e esclarecido antes do início do tratamento. Na consulta inicial, foram tiradas fotografias para possibilitar a observação de detalhes estéticos e auxiliar o plano de tratamento.

As fotografias registradas foram frontal intraoral, lateral intraoral direita e esquerda, sorriso frontal, sorriso lateral direita e esquerda, diagonal $\left(45^{\circ}\right)$ direita e esquerda e face frontal, o que possibilitaram observar face com proporções satisfatórias, sorriso médio, saúde periodontal satisfatória e padrões estéticos dentários. 

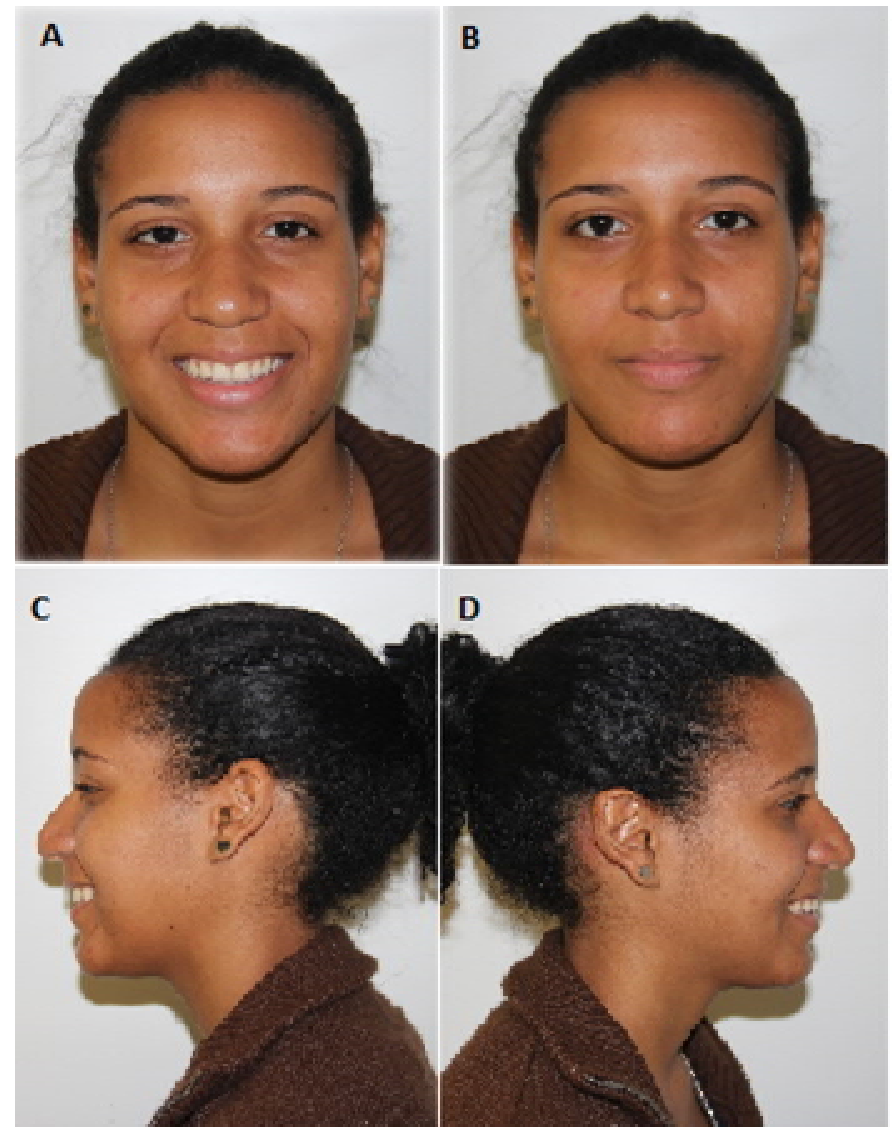

Figura 1 - A: Frontal de face sorrindo; B: Frontal de face lábios selados; C: Lateral esquerda da face sorrindo; D: Lateral direita da face sorrindo
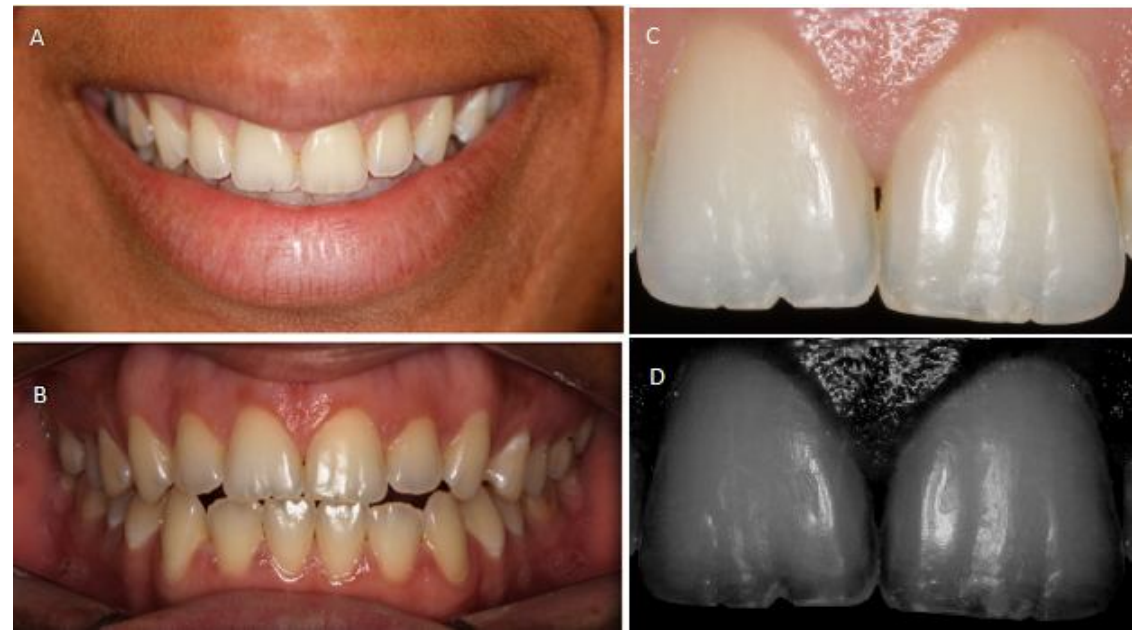

Figura 2 - A: Close sorriso frontal; B: intraoral frontal; C: Região incisal; D: Região incisal, em escala de cinza e mudança no contraste 
Após moldagem realizada com alginato (Dentsply, Petrópolis, Rio de Janeiro) e vazada com gesso tipo II (ASFER, São Caetano do Sul, São Paulo), foi obtido o modelo que possibilitou avaliação tridimensional, análise de detalhes de posicionamento, inclinações, formas dentais, bem como relações dos dentes em conjunto e com seus antagonistas.

Foram feitos os estudos e as devidas medidas dos dentes, através do paquímetro (Mitutoyo, Suzano, São Paulo), de acordo com as queixas do paciente e com as fotografias.

Para complementação do estudo do caso foi realizado um planejamento digital, em Power Point 2010, no qual foi observado simetria facial, simetria de sorriso e proporção entre dentes. Foi simulada a forma e tamanho final dos dentes, onde não foi observada necessidade de desgastes, contudo foram determinados aumentos (Figura 3):

- unidade 1.3 , aumento de $0,5 \mathrm{~mm}$ na face mesial e $0,5 \mathrm{~mm}$ em volume e $0,5 \mathrm{~mm}$ na mesial do bordo incisal.

- unidade 1.2, aumento de $0,5 \mathrm{~mm}$ na face distal e $1,5 \mathrm{~mm}$ no bordo incisal.

- unidade 1.1, aumento de 1,5 mm no bordo incisal.

- unidade 2.1, aumento de 1,0 mm no bordo incisal.

- unidade 2.2, aumento de 1,0 $\mathrm{mm}$ no bordo incisal e $0,5 \mathrm{~mm}$ na face distal.

- unidade 2.3, aumento de $0,6 \mathrm{~mm}$ na face mesial, $0,5 \mathrm{~mm}$ na face distal e $0,5 \mathrm{~mm}$ na mesial do bordo incisal.
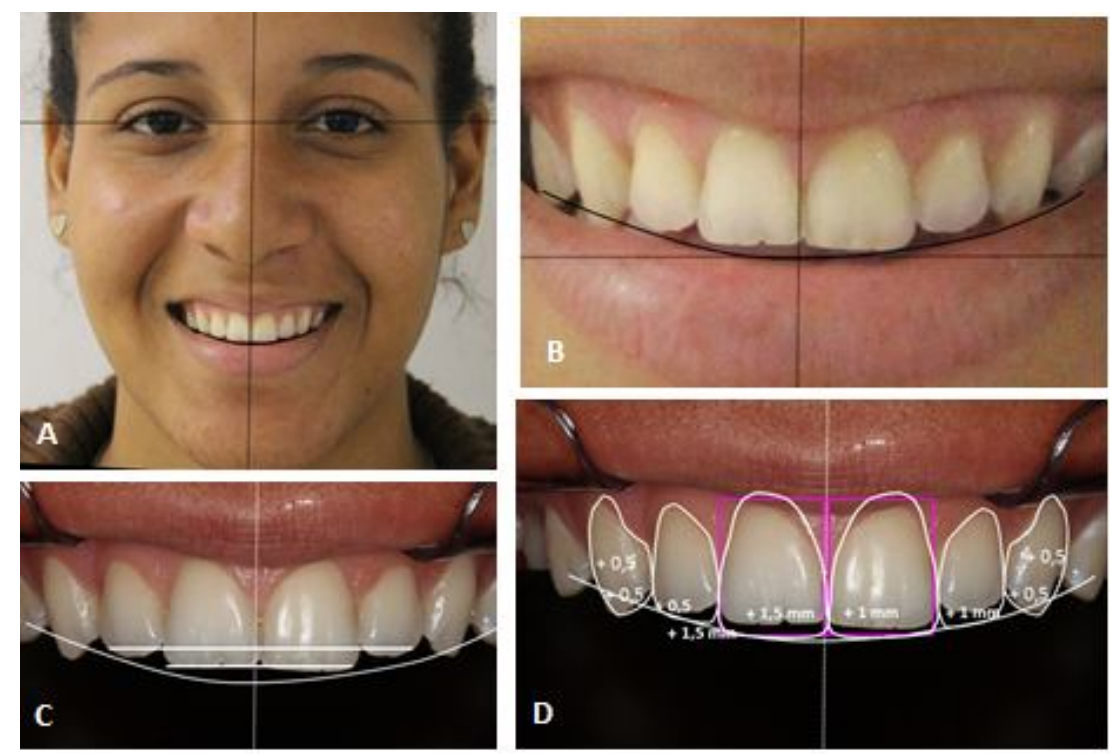

Figura 3 - A: simetria facial; B: curvatura incisal; C: relação altura do bordo incisal x curvatura incisal; D: planejamento digital (forma dos dentes, proporção altura x largura e quantidade de aumento

Revista Bahiana de Odontologia. 2013 out;4(2):147-157 http://www.bahiana.edu.br/revistas 
A partir destas análises preliminares, foi confeccionado o enceramento diagnóstico (Figura 4) preenchendo os diastemas na região dos incisivos laterais e caninos superiores de ambos os lados, além de um aumento na altura dos dentes na região de canino superior direito até o canino superior esquerdo. Posteriormente, foi confeccionado um guia de silicona de adição, consistência pesada (Elite HD, Zhermack, Moema, São Paulo) (Figura 5) para ensaio restaurador intraoral, a fim de propiciar a visualização prévia do resultado pela paciente.

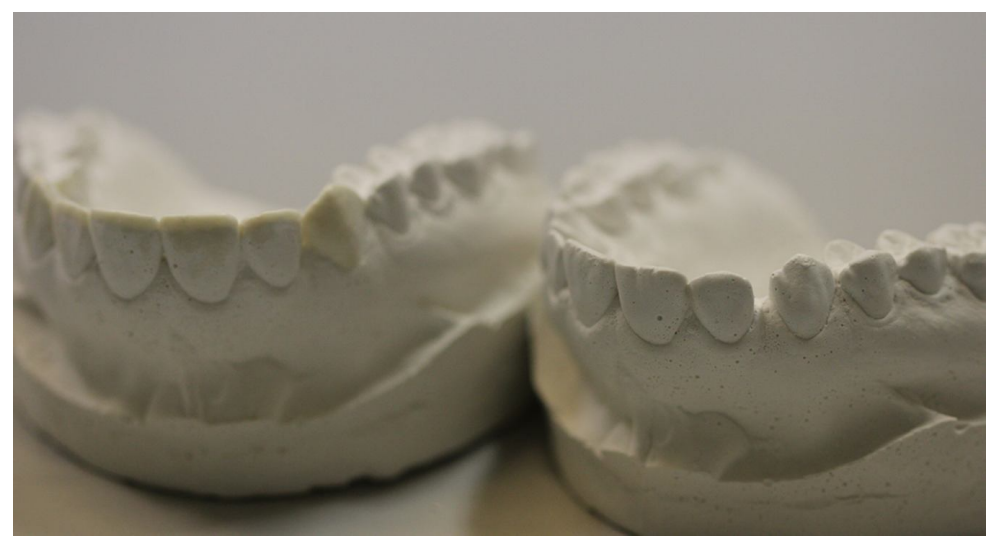

Figura 4 - Enceramento diagnóstico

A guia de silicona de adição, após recortada, foi levada em posição com uma resina bis-acrílica, cor A2, (Structur 2 SC, VOCO) (Figura 5). Após a polimerização do material, foram feitos ajustes com lâmina de bisturi, removendo excessos de áreas adjacentes às envolvidas no planejamento. O resultado desta etapa foi analisado criticamente por paciente e profissionais, levando ao consenso sobre o prognóstico, vantagens e limitações do tratamento. A partir daí, decidiu-se que a paciente será submetida à clareamento dental e posterior fechamento dos diastemas com resina composta, além do aumento dos incisivos centrais, laterais e caninos. 

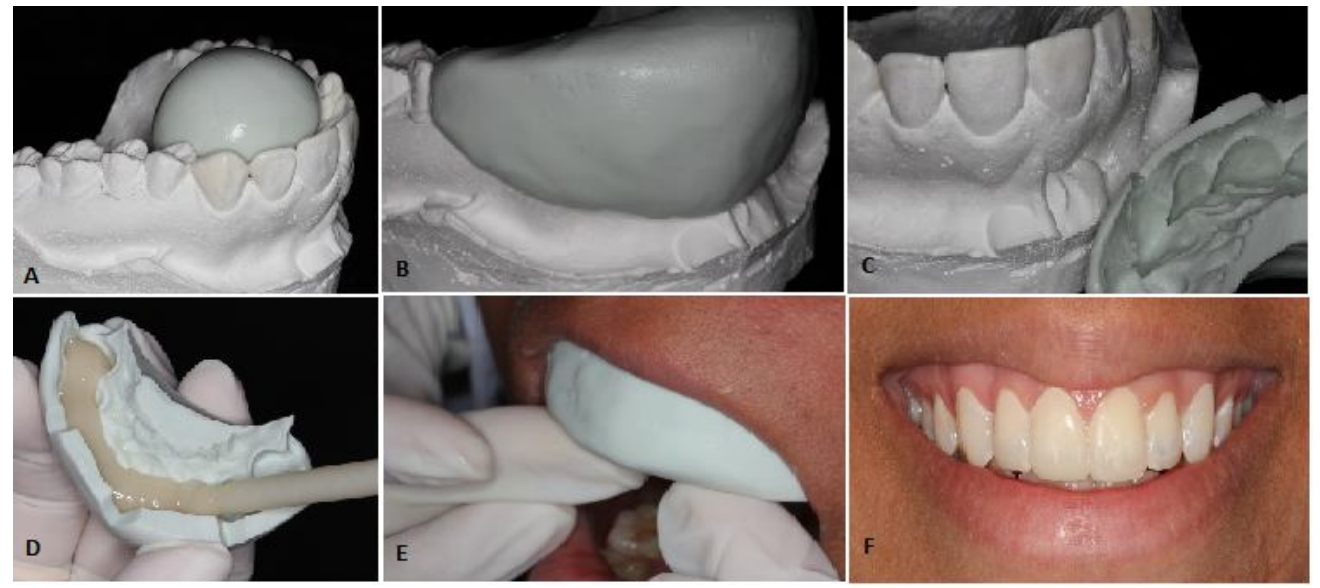

Figura 5 - A: Confecção da Guia de silicone; B: Posicionamento da Guia de silicona no modelo de estudo encerado; C: Guia de silicone; D: Adição de resina bis-acrílica na guia de silicone; E: Posicionamento em boca da guia de silicone; F: Ensaio restaurador intraoral

\section{DISCUSSÃO}

O aspecto do sorriso é resultado de um somatório de fatores. Dessa maneira, recursos de planejamento que possibilitem a visualização dos resultados previamente à execução, para que possíveis dificuldades sejam contornadas, favorecendo um melhor aspecto final, são fundamentais.

Dentre os recursos de planejamento disponíveis, encontram-se as fotografias, modelos de gesso, enceramento diagnóstico, ensaio restaurador intraoral e o planejamento digital. O uso de tais ferramentas torna possível uma correta avaliação estética para realização do planejamento, com ênfase aos aspectos relacionados à macro e microestética e as propriedades ópticas dos dentes.

A fotografia proporciona visualização de detalhes estéticos, auxilia na construção do plano de tratamento e é um meio de comunicação com o paciente e também com o laboratório. Os tipos de fotografias necessárias num planejamento integrado são: frontal intraoral, lateral intraoral, do sorriso, lábio em repouso, da face e lateral da face. Quando digitalizadas, estas imagens permitem profunda análise da macroestética e microestética, a partir de recursos como ampliação, mudança de contraste e brilho, entre outros (Figuras 1 e 2). Por outro lado, os modelos em estudo permitem uma visualização tridimensional dos dentes e tecidos adjacentes onde através deles podemos analisar detalhes sem ser necessária a presença do paciente (Figura 4$){ }^{(3,4)}$ 
As fotografias frontais de face, com o paciente sorrindo e com sorriso em repouso (Figuras 1A e 1B) demonstram aspectos da macroestética, como proporção entre os terços faciais, relação entre a distância interpupilar e o sorriso, e entre a linha média facial e a linha média dentária. Adicionalmente, fotografias frontais do sorriso permitem a análise do corredor bucal, tipo de lábio e tipo de sorriso.

A avaliação da linha média determina a simetria do arco já que representa uma linha imaginaria que divide os incisivos centrais superiores e inferiores. ${ }^{(5)}$ As linhas médias dentárias ideais (superior e inferior) devem ser coincidentes entre si e com a linha média facial. ${ }^{(6)}$ Desarmonias podem indicar necessidade de tratamento ortodôntico ou cirurgias. Após a colocação de linhas sobre as imagens frontais da face da paciente, verificou-se que a paciente apresenta simetria facial e coincidência da linha média facial e bucal. A linha interpupilar está perdendicular á linha média, dando uma aparência agradável à face (Figura $3 \mathrm{~A})$.

Associando as informações dos modelos de gesso, fotografias do sorriso frontal e intraoral em close-up (Figura 2A e 2B) é possível obter, em visão aproximada, referências sobre a linha do sorriso, alinhamento dental, forma e proporção entre dentes, contorno gengival, zênite gengival, cor e textura das unidades dentais, os quais são aspectos da microestética. No caso da paciente, após a análise das imagens frontais de sorriso e intraorais do arco superior, foi observada uma linha de sorriso média, dentes em formato levemente triangular, proporção entre dentes levemente desarmônica e presença de corredor bucal (Figura 3B, 3C e 3D). Estes detalhes devem ser analisados individualmente, para que restaurações estéticas ou re-anatomizações dentais sejam incorporadas com naturalidade e harmonia e os resultados sejam satisfatórios.

A linha do sorriso, ou curvatura incisal, é determinada por uma linha imaginaria, que passa tangente aos bordos incisais dos incisivos superiores e à ponta das cúspides dos caninos superiores, tendo grande influência na aparência estética do sorriso. ${ }^{(3)}$ A condição desejável é que a linha do sorriso seja convexa e acompanhe a curvatura do lábio inferior. Uma curvatura incisal côncava pode ser observada em idosos, ou em pessoas que apresentam alguma parafunção, ou que tenham hábitos oclusais nocivos, por exemplo, roer as unhas. ${ }^{(3)} \mathrm{Na}$ paciente avaliada, percebe-se que os incisivos superiores não tangenciam o lábio inferior, não possuindo convexidade adequada, havendo necessidade de aumento do bordo incisal dos mesmos, proporcionando maior harmonia do sorriso (Figura 3B e 3C). 
A presença de dentes alinhados e bem posicionados no arco dentário, contribuem, para a harmonia e equilíbrio estético do sorriso, permitindo uma transição gradual e suave no sentido antero-posterior e latero-central, quando a pessoa é vista a sorrir em posição frontal ou lateral, respectivamente. Quando há falta de alinhamento dentário gera-se uma tensão visual e perda no efeito de gradação entre os dentes, causando uma situação desfavorável do ponto de vista estético. ${ }^{(5)}$ Quanto ao alinhamento, a posição dos dentes é satisfatória, porém estão presentes diastemas nas proximais dos caninos e leve inclinação do incisivo lateral (unidade 2.2) para mesial. (figura de sorriso lateral)

Por meio da análise das fotografias intraorais e dos modelos de gesso também é relevante a observação da forma dos dentes,${ }^{(7)}$ que podem apresentar-se quadrados - onde as linhas externas são paralelas entre si; ovoides - linhas externas convergem para a cervical e incisal e os ângulos incisais são discretos; ou triangulares - linhas externas são convergentes para cervical e possuem ângulos incisais pronunciados. ${ }^{(3)}$ Este último formato foi considerado como predominante na paciente avaliada (Figura 2B).

A anatomia ou arquitetura e o tamanho dos dentes devem ser reproduzidos nas restaurações da forma mais realista possível. Nos homens, a proporção altura x largura do incisivo central superior assemelha-se a $85 \%$, nos incisivos laterais superiores, a $76 \%$ e nos caninos superiores, a 77\%. Para as mulheres esses valores alteram um pouco devido a tendência de dentes mais quadrados, com incisivo central superior entre 85-86\%, incisivos laterais superiores $79 \%$ e caninos superiores $81 \% .{ }^{(3)}$ Em relação às medidas abordadas no caso clínico, foi utilizada a proporção da altura x largura padrão ouro de $80 \%$.

A textura dos dentes também deve ser observada em fotografias intraorais, pois quanto maior a riqueza de detalhes, maior a reflexão de luz em diferentes direções, proporcionando aparência de dentes mais claros. ${ }^{(3)}$ A textura superficial caracterizada por linhas verticais (geralmente mais pronunciadas, e relacionadas com a formação de lóbulos dentários) e horizontais (que correspondem a linha de crescimento e tendem a desaparecer rapidamente com o desgaste da superfície vestibular do dente) ou pequenas depressões e, quando reproduzidas corretamente na restauração, dão uma maior aparência de dente natural. ${ }^{(3,8)}$

Quanto mais jovem o dente, mais caracterizado o mesmo será. Isto acontece pois, com o passar do tempo, devido ao contínuo desgaste de esmalte, há diminuição ou total perda da caracterização superficial. ${ }^{(3)}$ As imagens em close-up da paciente estudada revelam dentes 
caracterizados, com presença de mamelões e textura superficial pronunciada, principalmente nos incisivos centrais superiores e bordos translúcidos.

Para obter um sorriso agradável é necessário lançar mão de uma análise integrada da estética dental e do periodonto. Vários aspectos devem ser considerados no momento da avaliação, como por exemplo a condição periodontal, o biótipo periodontal, a linha do sorriso e exposição gengival, contorno e zênite gengival, a papila interdental e a coloração gengival. ${ }^{(9)}$

A exposição gengival nos indivíduos com sorriso alto é considerado normal se apresentar de 1 a $3 \mathrm{~mm}$. Se a medida for superior a $3 \mathrm{~mm}$ já será considerado sorriso gengival. ${ }^{(9)}$

O zênite é o limite mais apical da coroa do dente. A posição do zênite é determinada pela anatomia radicular, junção amelocementária e crista óssea. Nos incisivos centrais e caninos superiores localizam-se para distal em relação ao longo-eixo do dente e os incisivos laterais superiores coincide com o longo-eixo. ${ }^{(10)}$ Quando a altura e posição dos zênites encontram-se alteradas, existem opções cirúrgicas para tais correções, porém, no caso em questão, tais modificações não foram necessárias.

Ao final da coleta de todos estes importantes dados, foi realizado o enceramento diagnóstico. Esta etapa é fundamental por permitir a visualização preliminar do resultado esperado. ${ }^{(4,11)}$ Existem duas técnicas para a confecção do enceramento diagnóstico, a técnica aditiva e a regressiva, sua escolha depende da situação clínica apresentada. Na técnica aditiva a cera é adicionada ao modelo de estudo, sem ser necessário nenhum tipo de desgaste; seu uso é normalmente indicado em situações onde a análise indica necessidade de modificação dos volumes. Por outro lado, na técnica regressiva o desgaste será fundamental para a construção da anatomia dental, sendo em geral necessária naquelas situações onde a posição das unidades indica necessidade de realinhamento ${ }^{(12)}$ (Figura 4). Conforme visto anteriormente, na situação apresentada, os estudos indicaram necessidade de ampliação de volumes para correção das proporções dentais, e por este motivo, o enceramento aditivo foi executado.

Ao final, a partir do enceramento diagnóstico foi realizado o ensaio restaurador intraoral com resina bis-acrilica quimicamente ativada. Neste momento, foi possível analisar criticamente o resultado da adição de volume nos dentes antero-superiores da paciente. Apesar de existirem outros materiais para confecção deste ensaio, como as resinas compostas, ${ }^{(1)}$ a opção pela resina bis-acrilica como material se deveu à suas boas 
características como a facilidade de utilização e de manipulação, menor tempo de trabalho e boas propriedades mecânicas (dureza, módulo de elasticidade e resistência a flexão). ${ }^{(13)}$

\section{CONSIDERAÇÕES FINAIS}

O planejamento estético é um importante equipamento para o desenvolvimento de um efetivo tratamento dental, facilitando a comunicação do profissional com o paciente, o técnico do laboratório e outros profissionais, possibilitando uma maior aprovação do paciente no momento da finalização dos procedimentos.

Cada ferramenta tem sua função individual e em conjunto proporcionam um planejamento individualizado, respeitando as características de cada paciente. Para o desenvolvimento deste caso clínico foi seguido os seguintes passos:

$1^{\circ}$ Adequada anamnese, possibilitando a descoberta das expectativas do paciente diante do tratamento;

$2^{\circ}$ Execução de fotografias intra e extra orais;

$3^{\circ}$ Produção dos modelos de estudo;

$4^{\mathrm{o}}$ Estudo da micro e macro estética do paciente;

$5^{\circ}$ Planejamento digital e confecção do enceramento diagnóstico;

$6^{\circ}$ Ensaio restaurador intraoral.

A realização de um planejamento cuidadoso possibilita a previsibilidade do resultado, permitindo que possíveis problemas sejam contornados anteriormente à execução do tratamento. Com isso, os tratamentos passam a ter maior chance de sucesso estético e funcional.

\section{REFERÊNCIAS}

1. Watts A, Addy M. Tooth discoloration and staining: a review of the literature. British Dental Journal, 2001;190(6):309-16.

2. Fradeani M., Barducci G. Reabilitação estética em prótese fixa: tratamento protético. São Paulo: Quinterrence; 2009;2:0-116. 
3. Conceição EN. Restaurações estéticas: compósitos, cerâmicas e implantes. Porto alegre: Artmed; 2005.

4. Higashi $\mathrm{C}$ et al. Planejamento estético em dentes anteriores. In: MiyashitaE, Mello AT. Odontologia estética: planejamento e técnica. São Paulo: Artes Médicas Brasil; 2006. p. 13954.

5. Brum $\mathrm{C}$ et al. Estudo da proporção áurea em pacientes jovens classe II, divisão primeiro tratados ortodonticamente. Odonto, 2010;18(35):70-80.

6. Mondelli J. Estética e cosmética em clínica integrada restauradora. São Paulo: Quintessence; 2003.

7. Sterrett JD, Oliver T, Robinson F, Fortson W, Knaak B , Russell CM. Width/length ratios of normal clinical crowns of the maxillary anterior dentition in man. J. Clin Periodontol. 1999;26:153-157.

8. Correira A, Oliveira M, Silva M. Conceitos de estratificação nas restaurações de dentes anteriores com resina composta. Rev Port Estomatol Cir Malixofac, 2005;46(3):171-178.

9. Silva RC, Carvalho PFM, Joly JC. Planejamento estético em periodontia. In: Macedo, MCS, Baldaci Filho, R. Atualização clínica em odontologia. São Paulo: Artes Médicas; 2007. v. 1. [acesso em 28 set. 2013]. Disponível em:

http://www.implanteperio.com.br/publicacoes/capitulo-livro/planejamento-estetico-ciosp2007.pdf.

10. Myashita E, Fonseca AS. Odontologia estética: o estado da arte. São Paulo: Artes Médicas, 2004. p. 508-509.

11. Decurcio RA, et al._O uso do Mock-up na otimização e precisão do resultado da cirurgia plástica periodontal. Clín. int. j. braz. dent. Florianópolis, 2012;8(1):74-85.

12. Clavijo V, Kabbach W. Enceramento diagnóstico e sua aplicabilidade clínica. Clín. int. j. braz. dent. Florianópolis, 2012;8(2):146-159.

13. Kerby RE, Knobloch LA, Sharples S, Peregrina A. Mechanical properties of urethane and bis-acryl interim resin materials. J Prosthet Dent. 2013;110(1):21-8. 